\title{
Genetic inactivation of SARM1 axon degeneration pathway improves outcome trajectory after experimental traumatic brain injury based on pathological, radiological, and functional measures
}

\author{
Donald V. Bradshaw Jr. ${ }^{1,2}$, Andrew K. Knutsen ${ }^{3,4}$, Alexandru Korotcov ${ }^{3,4}$, Genevieve M. Sullivan ${ }^{2}$, \\ Kryslaine L. Radomski ${ }^{2,4}$, Bernard J. Dardzinski ${ }^{3,4}{ }^{*}$, Xiaomei $\mathrm{Zi}^{2}$, Dennis P. McDaniel ${ }^{5}$, and \\ Regina C. Armstrong ${ }^{1,2,4 \#}$ \\ ${ }^{1}$ Graduate Program in Neuroscience, \\ ${ }^{2}$ Department of Anatomy Physiology and Genetics \\ ${ }^{3}$ Department of Radiology and Radiological Sciences, \\ ${ }^{4}$ Center for Neuroscience and Regenerative Medicine, \\ ${ }^{5}$ Biomedical Instrumentation Center, \\ F. Edward Hebert School of Medicine, Uniformed Services University of the Health Sciences, \\ Bethesda, MD 20814 USA
}

Short title: Sarm1 deletion mitigates chronic TBI white matter atrophy

\section{\#Corresponding Author:}

Regina C. Armstrong, PhD

Department of Anatomy, Physiology and Genetics

Uniformed Services University of the Health Sciences

4301 Jones Bridge Rd., Bethesda, MD 20814

E-mail: regina.armstrong@usuhs.edu

${ }^{*}$ Current address:

Center for Scientific Review

National Institutes of Health, Bethesda, MD

\section{Author Contributions:}

D.V.B. and R.C.A. designed studies of chronic TBI in Sarm1 mice

K.L.R., D.V.B, and R.C.A. designed neuropathological analysis of tissue sections

D.V.B performed TBI surgical procedures

D.V.B., X.Z. and D.P.M performed neuropathological procedures

D.V.B. and R.C.A. performed neuropathological analyses

R.C.A, A.K.K, and A.K., B.J.D. and D.V.B. designed longitudinal MRI studies

A.K. and D.V.B. performed MRI studies

A.K.K. performed image processing for MRI studies

D.V.B., A.K.K., A.K. and R.C.A. analyzed MRI data

D.V.B. and G.M.S. performed behavioral studies

D.V.B., R.C.A, and G.M.S. designed and analyzed behavioral studies

R.C.A. secured funding for project

D.V.B. and R.C.A wrote the manuscript

All authors edited and approved the manuscript 


\section{ABSTRACT}

52 Traumatic brain injury (TBI) causes chronic symptoms and increased risk of neurodegeneration.

53 Axons in white matter tracts, such as the corpus callosum (CC), are critical components of neural

54 circuits and particularly vulnerable to TBI. Treatments are needed to protect axons from

55 traumatic injury and mitigate post-traumatic neurodegeneration. The Sarm1 gene is a central

56 driver of axon degeneration through a conserved molecular pathway. Sarm 1-/- mice with

57 knockout $(\mathrm{KO})$ of the Sarm1 gene enable genetic proof-of-concept testing of Sarm1 inactivation

58 as a therapeutic target. We evaluated Sarm1 deletion effects after TBI using a concussive model

59 that causes traumatic axonal injury and progresses to CC atrophy at 10 weeks, indicating post-

60 traumatic neurodegeneration. Sarm1 wild-type (WT) mice developed significant CC atrophy that

61 was reduced in Sarm1 KO mice. Using electron microscopy to quantify individual axons

62 demonstrated that Sarm1 KO preserved more intact axons and reduced damaged or demyelinated axons. MRI in live mice identified significantly reduced CC volume after TBI in Sarm1 WT mice that was attenuated in Sarm1 KO mice. MR diffusion tensor imaging detected reduced fractional anisotropy in both genotypes while axial diffusivity remained higher in Sarm1 KO mice. Immunohistochemistry revealed significant attenuation of CC atrophy, myelin loss, and neuroinflammation in Sarm1 KO mice after TBI. Functionally, TBI resulted in late-stage motor learning and sleep deficits that were ameliorated in Sarm1 KO mice. Based on these findings, Sarm1 inactivation can protect axons and white matter tracts to improve translational outcomes associated with CC atrophy and post-traumatic neurodegeneration.

71

72 Keywords: Chronic traumatic brain injury / SARM1; Sterile alpha and Toll/interleukin-1 receptor 73 containing motif 1 / white matter / corpus callosum atrophy / magnetic resonance imaging 


\section{INTRODUCTION}

Traumatic brain injury (TBI) results in long term disability in more severe cases and can cause persistent symptoms even in patients who receive a "mild" diagnosis [41, 64, 69]. TBI may also lead to post-traumatic neurodegeneration and increase the risk for co-morbid neurodegenerative diseases, such as Alzheimer's disease [15, 18, 30, 70]. In patients with moderate-severe TBI, diffuse axonal injury has been shown to predict the extent of posttraumatic neurodegeneration, based on MRI volumetric and diffusion tensor imaging (DTI) data [29]. The strongest relationship was found in central WM tracts, including the corpus callosum (CC). The CC is one of the main structures exhibiting atrophy across patients with complicated mild to severe TBI $[15,30]$. Furthermore, DTI tractography demonstrated disrupted fiber tract continuity in anterior CC regions after concussions while broad areas of disrupted tracts were found throughout the $\mathrm{CC}$ in patients with MRI findings of diffuse axonal injury [37]. Ex vivo MRI of a patient who died 26 days after TBI demonstrated that white matter tract disruptions detected by DTI correlated with neuropathological identification of axonal injury [66].

Long axons in white matter tracts are particularly vulnerable to damage in all forms of closed head TBI [10, 39, 55]. At an early stage of damage, axons can recover; once mechanical or molecular processes fragment the axon, then the distal axon irreversibly degrades by Wallerian degeneration $[31,36,53,54,65,89]$. The majority of axon damage is due to secondary mechanisms of damage that can cause axons to initiate Wallerian degeneration for weeks or more after the initial TBI event $[12,53,54]$. Irreversible axonal injury leads to disconnection between brain regions and disruption of neural circuits that may contribute to diverse TBI symptoms [32]. To improve outcomes for patients after TBI, research is needed to identify approaches to protect against axon degeneration and, further, to determine whether acute axon protection can reduce post-traumatic neurodegeneration.

Wallerian degeneration is an "active program of axon self-destruction" [73]. After injury or mitochondrial dysfunction, the SARM1 (sterile alpha and Toll/interleukin-1 receptor motif- 
102

containing 1) protein executes a highly conserved molecular axon death pathway [26, 47, 67, 80]. Injury releases SARM1 from an auto-inhibited state that is found in healthy axons [21, 73, 76]. Active SARM1 is a glycohydrolase which depletes nicotinamide adenine dinucleotide (NAD+) that is critical for energy stores in axons [17, 22, 25]. Loss of sufficient NAD+ leads to a cascade of axon destruction in which ionic imbalance leads to cytoskeletal and structural breakdown within the axon $[24,25,81]$.

Deletion of the Sarm1 gene provides a genetic "proof-of-concept" to examine the effects of long-term inactivation of this axon death pathway in chronic TBI and to evaluate the relationship of axonal injury to post-traumatic neurodegeneration. Knockout of Sarm1 in cultured human sensory neurons and in live mice reduced axon damage in trauma and in chemotherapyinduced models of peripheral neuropathy $[13,23,25,67]$. In multiple models of TBI, Sarm1 knockout $(\mathrm{KO})$ mice exhibited significantly reduced axon damage at acute and late phase time points $[34,51,56,92]$ Long-term studies of TBI in mice can model post-traumatic neurodegeneration that includes atrophy of the CC $[46,52,61]$. Initial results from our prior studies provided the first evidence that Sarm1 deletion may reduce both late stage axon damage and CC atrophy [51].

We now focus on CC atrophy as an important outcome measure of post-traumatic neurodegeneration. We evaluate the effect of Sarm1 deletion on axon damage, demyelination, and neuroinflammation on the progression of CC atrophy after closed head TBI. We use longitudinal MRI to detect changes in $\mathrm{CC}$ volume and white matter integrity in live mice, which provides a highly translational outcome measure of prolonged Sarm1 inactivation. To demonstrate whether the pathological effects of Sarm1 deletion have a meaningful impact on complex functions, we assess motor skill learning and sleep behavior. This experimental design provides a rigorous and translationally relevant screen of Sarm1 genetic inactivation as a potential therapeutic strategy for improving outcome measures after TBI. 


\section{MATERIALS AND METHODS}

\section{Mice}

of the Health Sciences and the National Institutes of Health Guide for the Care and Use of Laboratory Animals. Mice were socially housed with 2-5 mice per $35 \mathrm{~cm} \times 16.5 \mathrm{~cm} \times 18 \mathrm{~cm}$ cages containing enrichment objects. Mice were maintained on a standard $12 \mathrm{~h}$ cycle of daytime light (6:00-18:00). Sarm1-/- knockout (KO) mice, B6.129 × 1-Sarm1tm1Aidi/J (RRID:IMSR_JAX:018069) were originally obtained from The Jackson Laboratory. Sarm1 KO mice were crossed to C57BL/6J mice and then heterozygotes were bred to generate littermates for experiments. Sarm 1 is highly expressed in the brain; lack of detectable SARM1 protein in Sarm1 KO mice does not produce gross or microscopic brain pathology [27, 42]. The colony was maintained at Charles River Laboratories (Wilmington, MA) where ear biopsies were genotyped using a 3-primer allele specific PCR assay that targets the mutated Sarm1 region. Experimental mice were acclimated for 3 days after shipment and prior to the start of experiments. The total

$144 \quad(\mathrm{n}=75 ; 34$ male, 41 female $)$ littermates.

\section{TBI and Sham Procedures}

The TBI model has been characterized in our previous studies [51,58]. This concussive,

147 closed head injury model results in pathology under the impact site at bregma in the CC and the 148 adjacent cingulum $[52,79]$. Under $2 \%$ isoflurane anesthesia, 8-10 week old mice received a 149 single impact onto the skull at bregma using an ImpactOne stereotaxic impactor (Leica

150 Biosystems, Buffalo Grove, IL) with a 3-mm-diameter tip (velocity set at $4.0 \mathrm{~m} / \mathrm{s}$; depth of $1.5 \mathrm{~mm}$;

151 dwell time of $100 \mathrm{~ms})$. Sham mice received the same procedure without the impact. Righting

152 reflex demonstrated a significant injury effect of longer time to righting after TBI, as compared to 153 sham procedures, which did not differ with Sarm1 genotype or sex (Figure S1, Online Resource 
1). The predetermined study design criteria required exclusion of three mice for depressed skull fracture and/or impactor malfunction.

\section{Electron Microscopy}

Transmission electron microscopy (EM) was used to analyze axon and myelin subcellular structure, which can reveal a broad range of axon and myelin pathology $[52,58]$. Mice were anesthetized with ketamine/xylazine before transventricular cardiac perfusion with 4\% paraformaldehyde (Electron Microscopy Sciences, Hatfield, PA; Cat \#19210) and 2.5\% glutaraldehyde (Electron Microscopy Sciences; Cat \#16210) in $0.1 \mathrm{M}$ phosphate buffer. After overnight post-fixation, brains were cut into sagittal $(40 \mu \mathrm{m})$ sections using a Leica VT-1200 vibrating blade microtome (Leica Biosystems, Buffalo Grove, IL). Parasagittal sections were immersed in 2\% osmium tetroxide (OsO4; Electron Microscopy Sciences; Cat \#19100) infiltrated with Spurr epoxy resin (Electron Microscopy Sciences; Cat \#14300), flat-embedded and then polymerized at $70^{\circ} \mathrm{C}$ for $11 \mathrm{~h}$. Thin sections $(\sim 70 \mathrm{~nm})$ were cut on an Ultracut UC6

167 ultramicrotome (Leica Biosystems). Copper grids containing thin sections were post-stained for $20 \mathrm{~min}$ in $2 \%$ aqueous uranyl acetate (Electron Microscopy Sciences; Cat \#22400) and for 5 min

169 in Reynolds lead citrate (Reynolds, 1963).

\section{Quantification of sagittal CC width and electron microscopy analysis}

Cohorts consisted of Sarm1 WT sham ( $n=9 ; 4$ female, 5 male) and TBI $(n=8 ; 4$ female,

1724 male) along with Sarm1 KO sham ( $n=7 ; 3$ female, 4 male) and TBI ( $n=9 ; 5$ female, 4 male).

173 Mice were sacrificed at 10 weeks after sham or TBI procedure. Resin-embedded $40 \mu \mathrm{m}$ sagittal

174 sections approximately $200 \mu \mathrm{m}$ lateral to the midline were osmicated to stain myelin and imaged

175 in bright field on an Olympus IX-70 microscope. CC width was measured across the superior to

176 inferior borders in five locations at $\sim 100 \mu \mathrm{m}$ intervals across a $0.5 \mathrm{~mm}$ rostro-caudal region

177 centered under bregma. This region-of-interest (ROI) consistently exhibits traumatic axonal injury

178 in this TBI model, as evidenced by dispersed damaged axons among adjacent intact axons, and

179 matches the EM analysis performed in our previous work with this Sarm1 line of mice [51, 52]. 
Thin sections for EM analysis were then cut from within the CC ROI of the $40 \mu \mathrm{m}$ thick sections. The EM grids of sagittal thin sections were reviewed on a JEOL JEM-1011 transmission electron microscope (JEOL USA Inc., Peabody, MA) and images were acquired using an AMT XR50S-A digital camera (Advanced Microscopy Techniques, Woburn, MA). Images were taken at 5000x magnification and 8-10 images per animal were quantified for classification of axon and myelin pathology. For each image, a $17 \mu \mathrm{m} \times 12.5 \mu \mathrm{m}$ region defined the counting frame, within which >120 axons were quantified. All axons within the counting frame were counted. Axons partially crossing the top and right lines were included while those partially crossing the left and bottom line were excluded. Axons were classified as intact axons, de/unmyelinated axons, axons with abnormal mitochondria, or damaged axons $[51,52,58]$. Damaged axons were defined as axons with cytoskeletal disruption or axons with accumulated vesicles and debris. Mitochondria that appeared swollen encompassed $>50 \%$ of the area of the axon cross-section and were considered abnormal. De/unmyelinated axons were $>0.3 \mu \mathrm{m}$ in diameter and lacked detectable compact myelin, but otherwise appeared intact. Axons without myelin and with a diameter $<0.3 \mu \mathrm{m}$ were excluded as this axon size is typically unmyelinated in the CC of healthy adult mice [77]. TBI-induced demyelination was inferred when de/unmyelinated axon counts were significantly greater after TBI, as compared to sham. Myelin outfoldings were identified as myelin extending out from an axon and folding back onto itself to form double layered or redundant myelin [58] but were not significantly induced by TBI at this 10 week time point in mice of either genotype (data not shown). Additional images were taken at 10,000$15,000 x$ for illustration of pathological features.

\section{Magnetic resonance imaging (MRI) analysis of CC volume and microstructure}

MRI with multi-spin-echo, high resolution T2-weighted (T2-w) and diffusion tensor images (DTI) were acquired to assess the longitudinal effects of Sarm1 deletion in the CC ROI following

204 TBI. Longitudinal MRI studies were conducted in live mice with repeated scans of the same 205 mouse at baseline (prior to TBI) and at 3 days and 10 weeks post-TBI. Mice were scanned on a 

$\mathrm{cm}$ diameter gradient coil, $86 \mathrm{~mm}$ quadrature TX/RX coil, and Bruker Mouse head 4 channel receive coil array (Bruker BioSpin $\mathrm{GmbH}$, Reinstetten, Germany). Mice were anesthetized in an induction chamber with a mixture of $4 \%$ isoflurane in medical air and maintained with $1.5-1.75 \%$ isoflurane in medical air delivered by nose cone during the MRI procedures. Respiration rate (range 40-70 BPM, maintained by adjusting isoflurane concentration) and temperature were continuously monitored throughout the experiments. MRI slices were positioned using a sagittal

213 localizer so that coronal slices were oriented perpendicular to the length of the CC axis and each

214 brain was aligned with the midline crossing of the anterior commissure within in the same coronal slice $[78,83,91]$.

A whole brain T2 relaxation time map was generated from a two-dimensional rapid

217 acquisition with relaxation enhancement (2D RARE, coronal [33] using the following parameters:

$218 \mathrm{TR}=4000 \mathrm{~ms}$, echo time $(\mathrm{TE})=10,30,50,70,90,110 \mathrm{~ms}$, echo train length $(E T L)=2$, number of averages $\mathrm{NA}=4$, field of view (FOV) $14 \mathrm{~mm} \times 14 \mathrm{~mm}$, matrix 112x112, in-plane resolution 125 $\mu \mathrm{m} \times 125 \mu \mathrm{m}$, slice thickness $750 \mu \mathrm{m}$, number of slices $\mathrm{NS}=21$, no fat suppression, BW $36 \mathrm{kHz}$,

221 time 14:56 min. Additionally, a high resolution proton density weighted (PD-w) 3D MRI was

222 acquired to measure CC volumetrics using the following parameters: 3D RARE, TR $=2500 \mathrm{ms,}$

$223 \mathrm{TE}=30 \mathrm{~ms}, \mathrm{ETL}=8$, number of averages $\mathrm{NA}=1, \mathrm{FOV} 18 \mathrm{~mm} \times 14 \mathrm{~mm} \times 11 \mathrm{~mm}$, matrix

$224144 \times 112 \times 88,125 \mu \mathrm{m}$ isotropic resolution, no fat suppression, BW $36 \mathrm{kHz}$, time 55:25 min. DTI 225 data was acquired using the following parameters: 2D 2-shot echo planar imaging (EPI), TR= $2263000 \mathrm{~ms}, \mathrm{TE}=27, \mathrm{NA}=2,4 \mathrm{~B} 0$ and 30 non-collinear diffusion directions, $\mathrm{b}=600,1200 \mathrm{~s} / \mathrm{mm}^{2}$, $227 \delta=5 \mathrm{~ms}, \Delta=12 \mathrm{~ms}$, FOV $14 \mathrm{~mm} \times 14 \mathrm{~mm}$, in-plane resolution $175 \mu \mathrm{m} \times 175 \mu \mathrm{m}$, matrix 80x80, 228 slice thickness $750 \mu \mathrm{m}$, number of slices NS = 21, fat suppression, BW $300 \mathrm{kHz}$, time 12:48 min. T2-w images were converted to the NIFTI file format using a custom script in Matlab. A

230 bias field correction was performed on the $30 \mathrm{~ms}$ echo time image using the

231 N4BiasFieldCorrection command in the Advanced Normalization Toolkit (ANTs) [7]. The 
232

233

234

235

computed bias field was then applied to the other echo times (10, 50, 70, 90, $110 \mathrm{~ms})$. Estimates of T2-decay (T2) and amplitude ( $\mathrm{S}_{0}$ ) images (maps) were obtained using a nonlinear fit to the equation $\mathrm{S}_{\mathrm{i}}(\mathrm{TE})=\mathrm{S}_{0}{ }^{*} \exp (-\mathrm{TE} \mathrm{i} / \mathrm{T} 2)$, where $\mathrm{Si}$ is the signal intensity for echo time $\mathrm{TE}_{\mathrm{i}}$. The T2map and amplitude values were fed into a deep learning algorithm to create a brain mask [71, 78, 91]. Brain masks were manually corrected as needed. The ROI in the CC was drawn manually using VivoQuant software (inviCRO, Boston, MA). The CC ROI was defined as extending from the midline bilaterally to the point of ventral curvature in the external capsule (Online Resource 2), as in our previous studies [78, 91].

DTI were processed using the TORTOISE v3.2.0 software package $[62,68]$. Motion correction, eddy current correction, and EPI distortion correction were performed using the DIFFPREP function. Tensors were computed using a nonlinear tensor fit with RESTORE, and fractional anisotropy (FA), trace (TR), axial diffusivity (AD), and radial diffusivity (RD) were computed from the diffusion tensor images. An ROI was drawn manually in the CC within the slice under the impact site at bregma (Figure S2, Online Resource 1), and average CC values of FA, TR, AD, and RD were computed. TR did not show an effect of injury or genotype (data not shown).

To calculate volume change in the $\mathrm{CC}$ the $125 \mu \mathrm{m}$ isotropic 3D T2-weighted image slices were converted to NIFTI file format using a custom script in Matlab. A bias field correction was performed using the N4BiasFieldCorrection command in the Advanced Normalization Toolkit (ANTs) [7]. The brain mask from the multi-spin-echo T2 image set was transformed to the 3D T2w imaging using rigid registration and linear interpolation and thresholded at 0.5 to create a new binary mask. A template was created from the baseline scans using the antsMultivariateTemplateConstruction2.sh script [8]. Each image for each time point was then registered to the template using the nonlinear registration algorithm in ANTs [7]. Registration parameters were selected based on the approach described by Anderson et al. [3]. Voxel-wise maps of volume change were created using the CreateJacobianDeterminatelmage function in 
the ANTs toolkit. A ROI was drawn manually in the CC on each of seven $125 \mu \mathrm{m}$ coronal image slices (Online Resource 2) that encompassed the CC over the lateral ventricle and under the site of injury within the +0.5 and $-0.5 \mathrm{~mm}$ window relative to bregma. The average value of volume change was then computed. The statistical parametric mapping (SPM) v12 software was used to perform a voxel-wise analysis of the volume change images.

Immunohistochemistry

At 10 weeks following TBI or sham procedure, Sarm1 mice were perfused with $4 \%$ paraformaldehyde and brains cut as $14 \mu \mathrm{m}$-thick coronal cryosections for immunohistochemistry. Myelin was detected by immunolabelling for myelin oligodendrocyte glycoprotein (MOG; polyclonal mouse anti-MOG; 1:100; Millipore, Burlington, MA; Cat\# MAB5680, RRID: (GFAP; monoclonal mouse anti-GFAP; 1:500; Millipore, Burlington, MA; Cat\# MAB3402, RRID:AB_94844). Microglia/macrophages were identified using polyclonal rabbit antibody

271 against ionized calcium binding adaptor molecule 1 (IBA1; 1:500; Wako, Richmond, VA; Cat\#

272 019-19741, RRID:AB_839504). All tissue sections were counterstained with DAPI nuclear stain

273 (Sigma-Aldrich, St. Louis, MO; Cat\# D9542). after the TBI or sham procedure for Sarm1 WT sham $(n=7 ; 3$ female, 4 male) and TBI $(n=7 ; 3$ female, 4 male) along with Sarm $1 \mathrm{KO}$ sham $(n=6 ; 2$ female, 4 male) and TBI $(n=9 ; 5$ female, 3

278 male). Images within the CC ROI were acquired with a 10x objective on an Olympus IX-70

279 microscope using a SPOT RT3 camera. For quantification in coronal images, the CC ROI

280 extended from the midline laterally to under the peak of the cingulum at coronal levels between

$281+0.5$ and $-0.5 \mathrm{~mm}$ relative to bregma. The CC width (superior-inferior thickness) was measured

282 as the average of measurements taken at the midline and bilaterally at $\sim 200 \mu \mathrm{m}$ lateral to the 283 midline, under the peak of the cingulum, and $\sim 200 \mu \mathrm{m}$ lateral to the peak of the cingulum using 
MOG staining (Online Resource 2). ImageJ software was used to threshold fluorescence levels to quantify the area of immunolabeling above background [5, 51]. Images were also acquired with a $40 x$ objective to classify the morphology of IBA1 immunolabeled cells as resting or activated cells [58, 79, 91]. Quantification included 4-6 sections per mouse.

\section{Motor skill learning task}

The Miss-step wheel motor assay was performed using a protocol previously described [78]. The Miss-step wheel motor assay has been shown to engage the CC and be sensitive to changes in myelination $[35,57,59]$. From 8 to 10 weeks post TBI, mice were singly housed in home cages with a Miss-step running wheel equipped with an optical sensor to detect wheel revolutions (Mouse Miss-step Activity Wheel system, Cat\#80821, Lafayette Instruments,

294 Lafayette, IN). The Miss-step running wheels have 16 rungs missing from a standard wheel so that the remaining 22 rungs are distributed in an irregular interval pattern [35]. Mouse whiskers were clipped so that avoiding miss-steps was dependent on learning to follow the rung located on the prior step by bringing the hind paw forward to grasp the rung used by the forepaw [57]. were exported to a Microsoft Excel file every $24 \mathrm{hrs}$.

\section{Sleep/wake pattern}

Sleep pattern data was collected using a non-invasive automated scoring system (Signal Solutions LLC, Lexington, KY). Mice were single housed during the $8^{\text {th }}$ week following TBI or sham procedures for $72 \mathrm{hrs}$ and maintained on a standard $12 \mathrm{~h}$ cycle of daytime light (6:0018:00) with continuous data collection. A cage floor matt with piezoelectric sensors recorded 4 second epochs and used the $2-4 \mathrm{~Hz}$ breathing rhythm of mice to classify intervals of 30 seconds or more as asleep or awake [50]. This piezoelectric sensor system compares well with sleep data collected by visual observation and with electrophysiological discrimination of sleep/wake 
intervals, yet avoids the surgical procedures of electrophysiological techniques that could confound other assessments of TBI [60, 90].

\section{Statistical Analysis}

Sample sizes were estimated for $>80 \%$ power based on prior data in Sarm 1 and in C57BL/6 mice [51, 91] (Figures S2, S3, Online Resource 1). Mice were randomized to TBI/sham using the RAND function in Microsoft Excel. Investigators involved in data collection and analysis were blinded to genotype and injury condition until after the conclusion of the study. GraphPad Prism 8.0 software (RRID: SCR_002798) was used for statistical analysis and graphing. Bar graphs show means with standard error of the mean and symbols for individual mouse values. Two-way ANOVA was used to determine statistically significant differences when comparing by genotype and injury between groups. Repeated measures two-way ANOVA (RM ANOVA) was used to assess differences between genotypes over time in the longitudinal MRI analysis and within behavioral assessments. Corrections for multiple comparisons were done using Sidak's test. An alpha-level of $p$ value $<0.05$ was considered statistically significant.

\section{RESULTS}

\section{Sarm1 deletion protects against CC atrophy and axon-myelin pathology in chronic TBI}

The potential for Sarm1 inactivation to provide long term axon protection and/or to

327 mitigate post-traumatic neurodegeneration was first evaluated using transmission EM (Figure 1).

328 Before thin sectioning for EM, sagittal brain slices were osmium-stained to label myelin and examined with bright field microscopy to measure the CC width, which is a clinically relevant measure of white matter pathology in TBI and neurological diseases [19]. Both Sarm1 WT and

331 Sarm1 KO mice developed significant CC atrophy by 10 weeks after TBI, yet the extent of 332 atrophy was significantly less in the Sarm1 KO mice (Figure 1A). For in-depth analysis of the underlying pathology associated with changes in CC size, the tissue slices were then thin sectioned and imaged by transmission EM at subcellular resolution to examine individual 
myelinated axons within the same region of the CC. This quantification revealed a significant reduction of intact axons due to TBI in the Sarm1 WT mice that was rescued by Sarm1 deletion (Figure 1B).

Myelinated axons exhibit subcellular features that can distinguish intact axons from indicators of axon damage including swollen mitochondria, compacted cytoskeletal structure, accumulation of vesicles or debris, or loss of ensheathing myelin (Figure 1C-F). After the sham procedure, Sarm1 WT mice illustrate the healthy adult high density of myelinated axons in this anterior region of the CC (Figure 1G). As previously characterized in this concussive TBI model $[51,58]$, TBI results in damaged axons that are dispersed among adjacent intact axons and may also exhibit demyelination in Sarm1 WT mice (Figure 1H). Sarm1 KO mice exhibit normal appearing myelinated axons after the sham procedure and dispersed damaged axons after TBI (Figure 1I, J). Importantly, while both Sarm1 WT and Sarm1 KO mice exhibited axon damage after TBI, Sarm1 deletion significantly reduced the frequency of damaged axons (Figure 1K). Sarm1 deletion also normalized the frequency of TBI-induced mitochondrial pathology and demyelination (Figures 1L, M).

\section{Longitudinal in vivo MRI detects CC atrophy after TBI and attenuation by Sarm1 deletion}

Longitudinal MRI studies were conducted to advance the translational impact while further evaluating the effects of Sarm1 deletion on white matter integrity and CC atrophy. Each mouse was scanned prior to injury (i.e., baseline), and with follow up scans at acute and chronic stages after TBI. While DTI measures of white matter integrity have long been effectively used for analysis of adult mouse CC $[75,78]$, volume measurements have only recently been validated for quantification of atrophy and hypertrophy of the relatively small structures of adult mouse brains [3, 9]; Therefore, an initial cross-sectional in vivo study was conducted in C57BL/6 mice to optimize the MRI outcome measures. DTI analysis at 10 weeks after sham or TBI demonstrated significant changes after TBI in CC integrity while high resolution T2-w volumetrics 
361

362

detected significant CC atrophy that was validated by post-imaging neuropathology (Figure S2, Online Resource 1).

Longitudinal MRI studies were then conducted to compare Sarm1 WT and Sarm1 KO mice. Volumetric analysis showed significant atrophy of the CC between baseline and 10 weeks in Sarm1 WT mice (Figure 2A-C) that was attenuated in Sarm1 KO (Figure 2D-F). Across time points, DTI detected reduced white matter integrity after TBI based on reduced fractional anisotropy (FA) in both Sarm1 WT mice (Figure 2G, I) and Sarm1 KO mice (Figure 2H, I). Progressive decrease in FA was driven by decreased axial diffusivity (AD) at 3 days post-TBI (Figure 2J) and subsequent elevation of radial diffusivity (RD) at 10 weeks post-TBI (Figure 2K). Sarm1 genotype resulted in a significant effect of higher AD values in Sarm1 KO mice (Figure 2J).

\section{Immunohistochemistry shows reduced neuroinflammation in addition to with reduced CC} atrophy and myelin loss after TBI in mice with Sarm1 deletion.

Microglia and astrocyte responses have been linked to chronic white matter damage and atrophy $[40,44,45,49,58,79]$. Therefore, immunohistochemistry was used to evaluate CC atrophy and myelination (Figure 3A-D) and examine CC neuroinflammation in adjacent sections (Figure 4). CC width was measured in coronal sections with myelin immunolabeled for MOG and cytoarchitecture was evaluated using DAPI to stain nuclei (Figures 3E and S2, Online Resource 1). CC width was reduced at 10 weeks after TBI in comparison with sham mice (Figure 3E). Quantification of the pixel area of MOG immunoreactivity within the CC showed loss of myelin (Figure 3F), in agreement with the distributed demyelination observed by EM (Figure 1M). Sarm1 KO mice had significantly less CC atrophy and less myelin loss after TBI as compared to Sarm1 WT mice (Figure 3E, F).

Neuroinflammation was estimated by immunolabeling for reactive astrocytes and microglia using GFAP and IBA1, respectively (Figure 4). TBI resulted in more intense 
immunoreactivity of individual cells and an increase of overall immunolabeling for both GFAP and IBA1 (Figure 4A-G). Immunolabeling within the CC after TBI was reduced in Sarm1 KO mice for GFAP (Figure 4H) but not for IBA1 (Figure 4I). Further analysis to count the IBA1 immunolabeled cells within the CC revealed that Sarm1 deletion reduced microglia with either a resting state morphology (Figure 4J) or an activated morphology (Figure 4K) after TBI. Taken together, these results show that the reduction in CC atrophy and myelin loss seen in Sarm1 KO mice after TBI was accompanied by a reduced neuroinflammatory response.

\section{Sarm1 deletion improves functional outcome measures in chronic stage TBI}

Behavioral assessments targeting CC axons were selected to test whether the beneficial effects of Sarm1 deletion on TBI pathology translated to improved functional outcome measures. This TBI model did not cause overt symptoms at any time out through 10 weeks post-TBI. Therefore, an initial set of experiments in C57BL/6J mice evaluated two assays associated with CC axon-myelin pathology to determine whether either assay revealed deficits during this late phase of TBI. Miss-step wheel running is a motor skill task that engages CC axons and is sensitive to myelination status $[35,57,78]$. C57BL/6 mice showed a deficit in learning to run on the Miss-step wheels after TBI as compared to sham mice (Figure S3, Online Resource 1). C57BL/6 mice with CC pathology from experimental demyelination or repetitive mild exhibit social interaction deficits $\mathrm{TBI}[59,91]$. However, with the current single impact concussive model of TBI, C57BL/6 mice did not show social interaction deficits (Figure S4, Online Resource 1).

407 Based on the results of these assays, the Miss-step wheel task was selected for functional 408 assessment of TBI deficits in Sarm1 WT versus Sarm1 KO mice. 
413

414

415

416

parameters showed that Sarm $1 \mathrm{KO}$ mice ran on the wheels more times during the learning phase compared to Sarm1 WT mice (Figure 5B). Additionally, the cumulative distance traveled by Sarm1 KO mice during the learning phase is further for Sarm1 KO mice than for Sarm1 WT mice (Figure 5C).

Sleep behavior was selected in place of social interaction as a highly translational assessment for post-traumatic neurodegeneration (Figure 6). Sleep disorders are common in patients with chronic TBI and sleep patterns may contribute to neurodegeneration, particularly white matter degeneration [2, 63, 72]. Sleep data was analyzed for Sarm1 WT and Sarm1 KO mice during two light/dark cycles. In Sarm1 WT mice, TBI causes an overall difference from shams in the percent of time spent sleeping (Figure 6A). This difference was not found in Sarm1 KO mice (Figure 6B). More specifically, Sarm1 WT mice slept less during the lighted period that is the sleep phase for nocturnal mice (Figure 6C) without a change during the awake phase (Figure 6D). Sarm1 KO mice did not exhibit a sleep deficit after TBI, as compared to shams (Figure 6C, D).

\section{DISCUSSION}

The combined results from this study support a direct role of axonal injury in posttraumatic neurodegeneration and provide key pre-clinical evidence for TBI as a clinical indication for treatments to inhibit SARM1. Genetic inactivation of the gene for SARM1, which mediates a conserved axon degeneration pathway, had a beneficial effect on chronic white matter injury after TBI. Analysis of CC atrophy in longitudinal and crosssectional MRI studies was an effective outcome measure in mice that is a clinically useful measure of post-traumatic neurodegeneration. Complementary neuropathological techniques validated CC atrophy measures and identified beneficial effects from deletion of the Sarm1 gene on chronic stage axon damage, demyelination, and 
438

439

440

441

442

443

444

445

446

447

448

449

450

451

452

453

454

455

456

457

458

459

460

neuroinflammation. Importantly, Sarm1 deletion preserved healthy axons and prevented the progression of CC atrophy after TBI. Sarm1 deletion also ameliorated functional deficits which, together with the pathological and radiological benefits, indicate improved outcome trajectory after TBI.

Insights afforded by the subcellular detail of EM (Figure 1) address important gaps in the interpretation of long-term effects of Sarm1 deletion in experimental TBI. In the TBI model used in the current studies, EM data from the acute time point of 3 days [51] and chronic phase of 10 weeks post-TBI (Figure 1) are matched by scan time points in our longitudinal MRI of live mice (Figure 2). Prior studies in Sarm1 KO mice have shown significant axon protection in the acute (2 hours - 3 days) or chronic (2-6 months) phase after closed head TBI based on $\beta$-amyloid precursor protein ( $\beta$-APP) immunohistochemistry $[34,56]$ or using Thy1-YFP fluorescence to visualize axonal varicosities [51, 92]. The observation of subcellular details of axon and myelin ultrastructure afforded by EM informs a fuller interpretation of myelinated axon health. EM enables quantification of abnormal swollen mitochondria that are an early feature of axonal injury linked to Wallerian degeneration $[86,88]$ and have important implications for the SARM1 pathway $[36,47]$. Mitochondrial dysfunction challenges axon energy metabolism and creates a vulnerable axon state [36, 65]. Mitochondrial stress, trauma or other insults lead to loss of nicotinamide mononucleotide adenylyl-transferase 2 (NMNAT2), an NAD-synthesizing enzyme, in axons that depletes NAD+ and raises the level of its precursor nicotinamide mononucleotide (NMN) [20,47]. Low NAD+ and high NMN levels regulate release of auto-inhibition of SARM1 NADase activity, which further depletes NAD+ and axon energy stores [11, 21, 38, 73, 76]. Axons with swollen 
461 mitochondria were significantly increased at 3 days after TBI in Sarm1 WT and Sarm1

$462 \mathrm{KO}$ mice [51]. Compared to this acute data, axons with swollen mitochondria were less

463 frequent at 10 weeks but still significantly increased after TBI in Sarm1 WT mice (Figure

464 1), which indicates the potential for continued activation of SARM1.

EM identifies damaged axons based on breakdown and compaction of the

466 cytoskeleton and/or accumulated vesicles due to impaired axonal transport (Figure 1).

467 The frequency of damaged axons and the effect of Sarm1 deletion is very similar at the

46810 week time point (Figure 1) as compared to 3 days post-TBI [51]. The slow clearance

469 of degenerating axons in the CNS may play a part in this result [85]. Alternatively,

470 surviving axons may succumb to insults in a late phase after TBI and Sarm1 deletion

471 may have on ongoing benefit across acute and chronic time points. For example, axons

472 continue to initiate Wallerian degeneration long after stretch injury that models TBI forces

473 [54]. Clinically, persistent neuroinflammation in white matter is associated with axon

474 damage in the CC in human postmortem cases several years after TBI [39].

475 Finally, EM is the gold standard to quantify demyelination, i.e. loss of the myelin

476 sheath around otherwise healthy axons. TBI induced demyelination in Sarm1 WT mice

477 that was not found in Sarm1 KO mice at 10 weeks post-TBI or sham procedures (Figure

478 1). Sarm1 is expressed mainly in neurons but a low level of expression in

479 oligodendrocyte lineage cells could have a role in the lack of TBI-induced demyelination

480 in Sarm1 KO mice [43]. However, the effect of Sarm1 deletion may not be an

481 autonomous effect in myelinating oligodendrocytes as zebrafish studies have shown a

482 glioprotective effect of Sarm1 deletion is dependent on axon protection [82]. Loss of 
484 desynchronize neural circuits; myelin also protects from insults and provides trophic

485 support to axons [6]. Therefore, demyelination that is resolved by Sarm1 deletion may

486 have ongoing benefit at 10 weeks post-TBI that can impact axon health and neural circuit 487 function.

The current study is the first to use translational measures of MRI with CC atrophy

489 to evaluate the effects of Sarm1 deletion after TBI. Based on histological measures of

490 CC width, our prior studies in this TBI model did not detect significant CC atrophy at the

4913 day, 2 week, or 6 week time points [51,58]. In contrast, significant CC atrophy was

492 evident by histological measures at the longer time point of 8 weeks post-TBI [51, 52]

493 and now at 10 weeks post-TBI (Figures 1A, 3E and S2, Online Resource 1). At this 10

494 week time point, these histological measures validate the changes in CC volume in live

495 mice determined by quantitative MRI (Figure 2C and S2, Online Resource 1).

496 Significantly, the histological and MRI measures corroborate attenuation of CC atrophy in

497 Sarm1 KO mice as compared to Sarm1 WT mice (Figures 1A, 2F, 3E). These

498 longitudinal MRI studies also analyzed DTI FA as a measure of CC microstructure [87,

499 90]. Reduced FA values indicated an injury effect at 3 days and at 10 weeks after TBI, as

500 compared to baseline (Figure 2I-K and S2, Online Resource 1). A main effect of Sarm1

501 genotype was only observed for the AD parameter (Figure 2J) that is most often

502 associated with axonal anisotropy within white matter voxels. Interpreting reduced AD as

503 an indicator of axon pathology and increased RD as an indicator of myelin pathology can

504 be useful when each pathology predominates [75, 89]. The presence of simultaneous

505 pathology of axon damage and demyelination is more difficult to detect and interpret

506 using DTI, and is further complicated in the presence of significant edema or 
neuroinflammation [87, 89]. Histological measures confirm the presence of simultaneous axon damage, demyelination, and neuroinflammation at 10 weeks after TBI along with significant reduction of each pathology with Sarm1 deletion (Figures 1, 3, 4), which is not fully appreciated using the DTI parameters (Figure 2I-K).

Pathological and structural benefits of Sarm1 deletion were complemented by behavioral studies that show amelioration of functional deficits (Figures 5,6 ). Our results extend beyond previous reports in Sarm1 KO mice to now examine complex behaviors during the chronic phase post-TBI. Studies in a weight drop model of TBI used the neurological severity score battery of simple tasks from 2 hours through 4 weeks postTBI and found reduced deficits in Sarm1 KO versus Sarm1 WT mice only during the first week [34]. Studies of repetitive TBI showed normalization toward sham responses in Sarm1 KO for motor performance and memory deficits during the first week and context fear discrimination at 4 weeks [56]. With assessments conducted beyond 8 weeks postTBI, Sarm1 deletion reduced deficits during motor learning (Figure 5) and normalized the time spent sleeping (Figure 6). The Miss-step wheel running system (Figure 5) and the piezoelectric sleep system (Figure 6) use automated multi-day continuous data collection of spontaneous complex behaviors, which may be advantageous for analysis of subtle deficits after TBI.

Limitations of the experimental design should be considered in the interpretation of the results. The Sarm1 KO mice have been backcrossed to the C57BL/6 strain but may harbor genes associated with the embryonic stem cell origin in the 129 background strain [84]. However, the findings regarding axon degeneration in this line of Sarm1 KO mice have been confirmed in additional Sarm1 KO mice generated using CRISPR 
530 technology [84]. In addition, all experiments in the current study used Sarm1 WT and

531 Sarm1 KO littermates to minimize the variability due to genetic background. The

532 assessment of sleep disorders after TBI used a non-invasive screening approach to

533 avoid surgical procedures and electrode placement through skull burr holes that would

534 be required for electrophysiology. The sleep behavior in the Sarm1 KO mice appears

535 normalized to the sham sleep pattern but electrophysiological recordings would be

536 needed for a more in-depth comparison of sleep architecture. Finally, the

537 neuropathological and radiological analyses focused on white matter, and specifically the

538 CC. Further studies would be of interest to better understand the relationship of axon

539 damage and the progression of white matter pathology relative to broader neural circuits

540 and gray matter pathology, including analysis of synapse loss that is associated with

541 chronic neuroinflammation after TBI [1].

\section{CONCLUSIONS}

These results demonstrate that genetic inactivation of Sarm1 improves the

545 outcome trajectory after TBI based on pathological, radiological, and functional

546 measures. These studies advance strategies to develop TBI treatments for axon damage

547 by demonstrating a genetic proof-of-concept of the long-term benefit of Sarm1 deletion.

548 The therapeutic potential of SARM1 inhibitors has drawn intense interest and a small

549 molecule inhibitor of SARM1 has already been developed that recapitulates in vitro

550 aspects of the Sarm1 KO phenotype [36, 48, 74]. The current studies also highlight CC

551 atrophy as an important outcome measure of white matter degeneration after TBI in mice

552 that may have translational relevance as a biomarker for clinical studies [28]. White 
553 matter degeneration may be a tractable therapeutic target for TBI and chronic traumatic

554 encephalopathy, with potential application to other neurodegenerative diseases including 555 Alzheimer's disease and multiple sclerosis [4, 14, 16, 70].

\section{Acknowledgements}

558 The authors thank Dr. Christina Marion and Dr. Krystal Schaar Valenzuela for technical

559 advice. We appreciate the support of the Center for Neuroscience and Regenerative

560 Medicine Preclinical Models Core, Translational Imaging Core, and Translational

561 Therapeutics Core and the Biomedical Instrumentation Center at the Uniformed Services

562 University. These studies were funded by the U.S. Department of Defense and the

563 Uniformed Services University through the UCSF-USUHS Partnership: Brain Injury and

564 Disease Prevention, Treatment, and Research and the Center for Neuroscience and

565 Regenerative Medicine. The authors declare no competing financial interests. Opinions

566 are those of the authors and do not represent the University, the Department of Defense,

567 or the federal government. 
Lozano D, Gonzales-Portillo GS, Acosta S, de la Pena I, Tajiri N, Kaneko Y, Borlongan CV (2015) Neuroinflammatory responses to traumatic brain injury: etiology, clinical consequences, and therapeutic opportunities. Neuropsychiatr Dis Treat 11: 97-106 Doi 10.2147/ndt.S65815

50 Mang GM, Nicod J, Emmenegger Y, Donohue KD, O'Hara BF, Franken P (2014) Evaluation of a piezoelectric system as an alternative to electroencephalogram/ electromyogram recordings in mouse sleep studies. Sleep 37: 1383-1392 Doi 10.5665/sleep.3936

51 Marion CM, McDaniel DP, Armstrong RC (2019) Sarm1 deletion reduces axon damage, demyelination, and white matter atrophy after experimental traumatic brain injury. Exp Neurol 321: 113040 Doi 10.1016/j.expneurol.2019.113040

52 Marion CM, Radomski KL, Cramer NP, Galdzicki Z, Armstrong RC (2018) Experimental Traumatic Brain Injury Identifies Distinct Early and Late Phase Axonal Conduction Deficits of White Matter Pathophysiology, and Reveals Intervening Recovery. The Journal of Neuroscience 38: 8723-8736 Doi 10.1523/jneurosci.0819-18.2018

53 Marmarou CR, Walker SA, Davis CL, Povlishock JT (2005) Quantitative analysis of the relationship between intra- axonal neurofilament compaction and impaired axonal transport following diffuse traumatic brain injury. J Neurotrauma 22: 1066-1080 Doi 10.1089/neu.2005.22.1066

54 Maxwell WL, Bartlett E, Morgan H (2015) Wallerian degeneration in the optic nerve stretch-injury model of traumatic brain injury: a stereological analysis. J Neurotrauma 32: 780-790 Doi 10.1089/neu.2014.3369

55 Maxwell WL, Povlishock JT, Graham DL (1997) A mechanistic analysis of nondisruptive axonal injury: a review. J Neurotrauma 14: 419-440 Doi 10.1089/neu.1997.14.419

56 Maynard ME, Redell JB, Zhao J, Hood KN, Vita SM, Kobori N, Dash PK (2020) Sarm1 loss reduces axonal damage and improves cognitive outcome after repetitive mild closed head injury. Exp Neurol 327: 113207 Doi 10.1016/j.expneurol.2020.113207

57 McKenzie IA, Ohayon D, Li H, de Faria JP, Emery B, Tohyama K, Richardson WD (2014) Motor skill learning requires active central myelination. Science 346: 318-322 Doi 10.1126/science.1254960

58 Mierzwa AJ, Marion CM, Sullivan GM, McDaniel DP, Armstrong RC (2015) Components of myelin damage and repair in the progression of white matter pathology after mild traumatic brain injury. J Neuropathol Exp Neurol 74: 218-232 Doi 10.1097/NEN.0000000000000165

59 Mierzwa AJ, Zhou YX, Hibbits N, Vana AC, Armstrong RC (2013) FGF2 and FGFR1 signaling regulate functional recovery following cuprizone demyelination. Neurosci Lett 548: 280-285 Doi 10.1016/j.neulet.2013.05.010

60 Mountney A, Boutté AM, Cartagena CM, Flerlage WF, Johnson WD, Rho C, Lu XC, Yarnell A, Marcsisin S, Sousa Jet al (2017) Functional and Molecular Correlates after Single and Repeated Rat Closed-Head Concussion: Indices of Vulnerability after Brain Injury. J Neurotrauma 34: 27682789 Doi 10.1089/neu.2016.4679

61 Mouzon B, Bachmeier C, Ojo J, Acker C, Ferguson S, Crynen G, Davies P, Mullan M, Stewart W, Crawford F (2019) Chronic White Matter Degeneration, but No Tau Pathology at One-Year PostRepetitive Mild Traumatic Brain Injury in a Tau Transgenic Model. J Neurotrauma 36: 576-588 Doi 10.1089/neu.2018.5720

62 Mustafa Okan Irfanoglu AN, Jeffrey Jenkins, Carlo Pierpaoli (2018) TORTOISEv3:Improvements and New Features of the NIH Diffusion MRI Processing Pipeline. ISMRM 25th annual meeting, City 63 Nedergaard M, Goldman SA (2020) Glymphatic failure as a final common pathway to dementia. Science 370: 50-56 Doi 10.1126/science.abb8739

64 Nelson LD, Temkin NR, Dikmen S, Barber J, Giacino JT, Yuh E, Levin HS, McCrea MA, Stein MB, Mukherjee Pet al (2019) Recovery After Mild Traumatic Brain Injury in Patients Presenting to US Level I Trauma Centers: A Transforming Research and Clinical Knowledge in Traumatic Brain Injury (TRACK-TBI) Study. JAMA Neurol 76: 1049-1059 Doi 10.1001/jamaneurol.2019.1313 
82381 Summers DW, Gibson DA, DiAntonio A, Milbrandt J (2016) SARM1-specific motifs in the TIR domain enable NAD+ loss and regulate injury-induced SARM1 activation. Proc Natl Acad Sci U S A 113: E6271-E6280 Doi 10.1073/pnas.1601506113

82 Tian W, Czopka T, López-Schier H (2020) Systemic loss of Sarm1 protects Schwann cells from chemotoxicity by delaying axon degeneration. Commun Biol 3: 49 Doi 10.1038/s42003-020-07769

83 Tobin JE, Xie M, Le TQ, Song SK, Armstrong RC (2011) Reduced axonopathy and enhanced remyelination after chronic demyelination in fibroblast growth factor 2 (Fgf2)-null mice: differential detection with diffusion tensor imaging. J Neuropathol Exp Neurol 70: 157-165 Doi 10.1097/NEN.0b013e31820937e4

84 Uccellini MB, Bardina SV, Sánchez-Aparicio MT, White KM, Hou YJ, Lim JK, García-Sastre A (2020) Passenger Mutations Confound Phenotypes of SARM1-Deficient Mice. Cell Rep 31: 107498 Doi 10.1016/j.celrep.2020.03.062

85 Vargas ME, Barres BA (2007) Why is Wallerian degeneration in the CNS so slow? Annu Rev Neurosci 30: 153-179 Doi 10.1146/annurev.neuro.30.051606.094354

86 Vial JD (1958) The early changes in the axoplasm during wallerian degeneration. J Biophys Biochem Cytol 4: 551-555 Doi 10.1083/jcb.4.5.551

87 Wang X, Cusick MF, Wang Y, Sun P, Libbey JE, Trinkaus K, Fujinami RS, Song SK (2014) Diffusion basis spectrum imaging detects and distinguishes coexisting subclinical inflammation, demyelination and axonal injury in experimental autoimmune encephalomyelitis mice. NMR Biomed 27: 843-852 Doi 10.1002/nbm.3129

88 Williams PR, Marincu B-N, Sorbara CD, Mahler CF, Schumacher A-M, Griesbeck O, Kerschensteiner M, Misgeld T (2014) A recoverable state of axon injury persists for hours after spinal cord contusion in vivo. Nature Communications 5: 5683 Doi 10.1038/ncomms6683

89 Xie M, Tobin JE, Budde MD, Chen Cl, Trinkaus K, Cross AH, McDaniel DP, Song SK, Armstrong RC (2010) Rostrocaudal analysis of corpus callosum demyelination and axon damage across disease stages refines diffusion tensor imaging correlations with pathological features. J Neuropathol Exp Neurol 69: 704-716 Doi 10.1097/NEN.0b013e3181e3de90

90 Yaghouby F, Donohue KD, O'Hara BF, Sunderam S (2016) Noninvasive dissection of mouse sleep using a piezoelectric motion sensor. J Neurosci Methods 259: 90-100 Doi 10.1016/j.jneumeth.2015.11.004

91 Yu F, Shukla DK, Armstrong RC, Marion CM, Radomski KL, Selwyn RG, Dardzinski BJ (2017) Repetitive Model of Mild Traumatic Brain Injury Produces Cortical Abnormalities Detectable by Magnetic Resonance Diffusion Imaging, Histopathology, and Behavior. J Neurotrauma 34: 13641381 Doi 10.1089/neu.2016.4569

92 Ziogas NK, Koliatsos VE (2018) Primary Traumatic Axonopathy in Mice Subjected to Impact Acceleration: A Reappraisal of Pathology and Mechanisms with High-Resolution Anatomical Methods. J Neurosci 38: 4031-4047 Doi 10.1523/JNEUROSCI.2343-17.2018 


\section{FIGURE LEGENDS}

\section{Figure 1. Sarm1 deletion reduces corpus callosum atrophy and axon pathology at 10}

weeks post-TBI. A: Sarm1 deletion attenuates CC atrophy that develops after TBI. B: Sarm1

deletion preserves intact, myelinated axons after TBI. C-F: Representative images of

pathological features from high resolution electron microscopy in CC sagittal sections. C:

Example from a Sarm1 WT sham mouse to show the cytoskeleton, mitochondria and surrounding myelin sheath of intact axons in contrast with a rare damaged axon with a densely compacted cytoskeleton (blue arrow). D: Sarm1 WT TBI mouse example of an axon with an abnormally large mitochondrion (red arrow) and an axon with a typical mitochondrion (red asterisk). Mitochondria were considered abnormal when swollen to $>50 \%$ of the axon area. E:

Demyelinated axon (yellow arrow) lacking ensheathing myelin but with otherwise intact cytoskeleton in a Sarm1 WT TBI mouse. F: Damaged axon (blue arrow) with vesicle accumulation and cytoskeletal breakdown in a Sarm1 KO TBI mouse. G-J: Representative electron microscopy images from CC sagittal sections for Sarm1 WT sham $(G)$ and TBI $(H)$ mice in comparison with Sarm1 KO sham (I) and TBI (J) mice. Arrows identify examples of damaged axons with accumulated vesicles and/or compacted cytoskeleton (blue), abnormal mitochondria (red), or demyelination (yellow). K-M: Quantification of axon and myelin pathology at 10 weeks after TBI or sham procedure. Sarm1 deletion reduces chronic stage axon damage (K), normalizes mitochondria morphology (L), and eliminates TBI-induced demyelinated component of de/unmyelinated axons (M). Sarm1 WT: $n=9$ sham, $n=8$ TBI. Sarm 1 KO: $n=7$ sham, $n=9$ TBI. ns = not significant. C-F, scale bars $=0.5 \mu \mathrm{m}$. G-J, scale bars $=1 \mu \mathrm{m}$. 

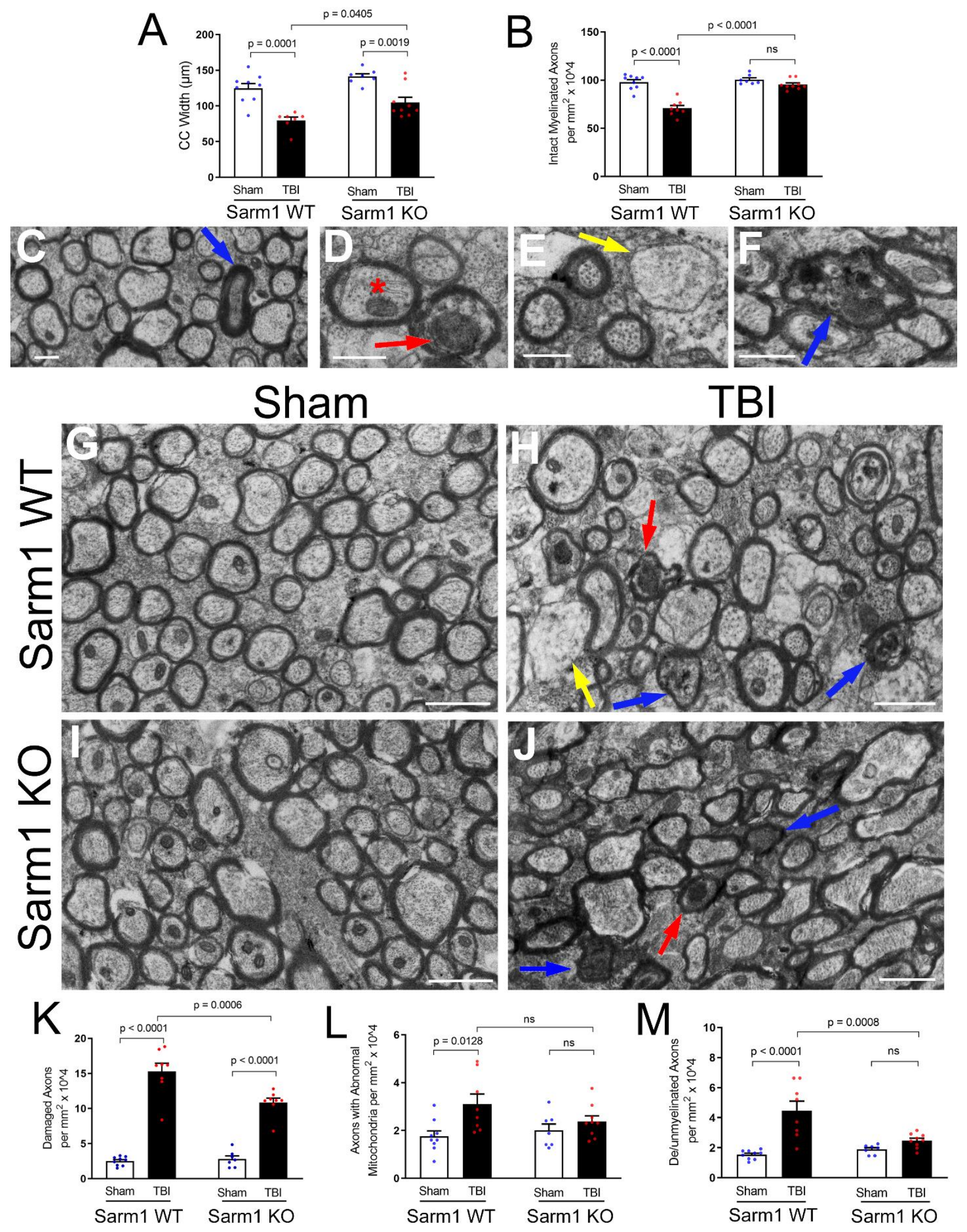

Sarm1 WT mice. T2-weighted images showing the coronal view at the level of the impact site at baseline (A, before surgery) and at 10 weeks (B) post-TBI/sham procedures. Quantification of

895 volume change in CC regions under the impact site (C). D-F: In Sarm1 KO mice, CC atrophy is 896 not detected in representative T2-weighted images (D, E) or based on changes in CC volume 897 (F). G-H: Direction encoded color images of diffusion tensor imaging (DTI) from a longitudinal $898 \mathrm{MRI}$ series at baseline $(\mathrm{BL})$ and again at 3 days and 10 weeks post-TBI or sham procedures. 899 Colors represent fiber directions as red (medial-lateral), blue (anterior-posterior), and green 900 (superior-inferior). I-K: Quantification of DTI measures reveals a chronic progression of CC 901 pathology following TBI. Fractional anisotropy (FA) significantly decreases over time following $902 \mathrm{TBI}(\mathrm{I})$. The acute change is driven by a decrease in axial diffusivity (AD) between baseline and 3 903 days (J). Sarm1 KO mice have significantly higher AD values than Sarm1 WT mice (J). The FA 904 at 10 weeks corresponds with a delayed increase in radial diffusivity (RD) in Sarm1 KO mice (K). 905 Arrows indicate medial CC regions. Sarm1 WT: $\mathrm{n}=10 \mathrm{TBI}$; Sarm1 KO: $\mathrm{n}=15 \mathrm{TBI}$. ns = not 906 significant. 

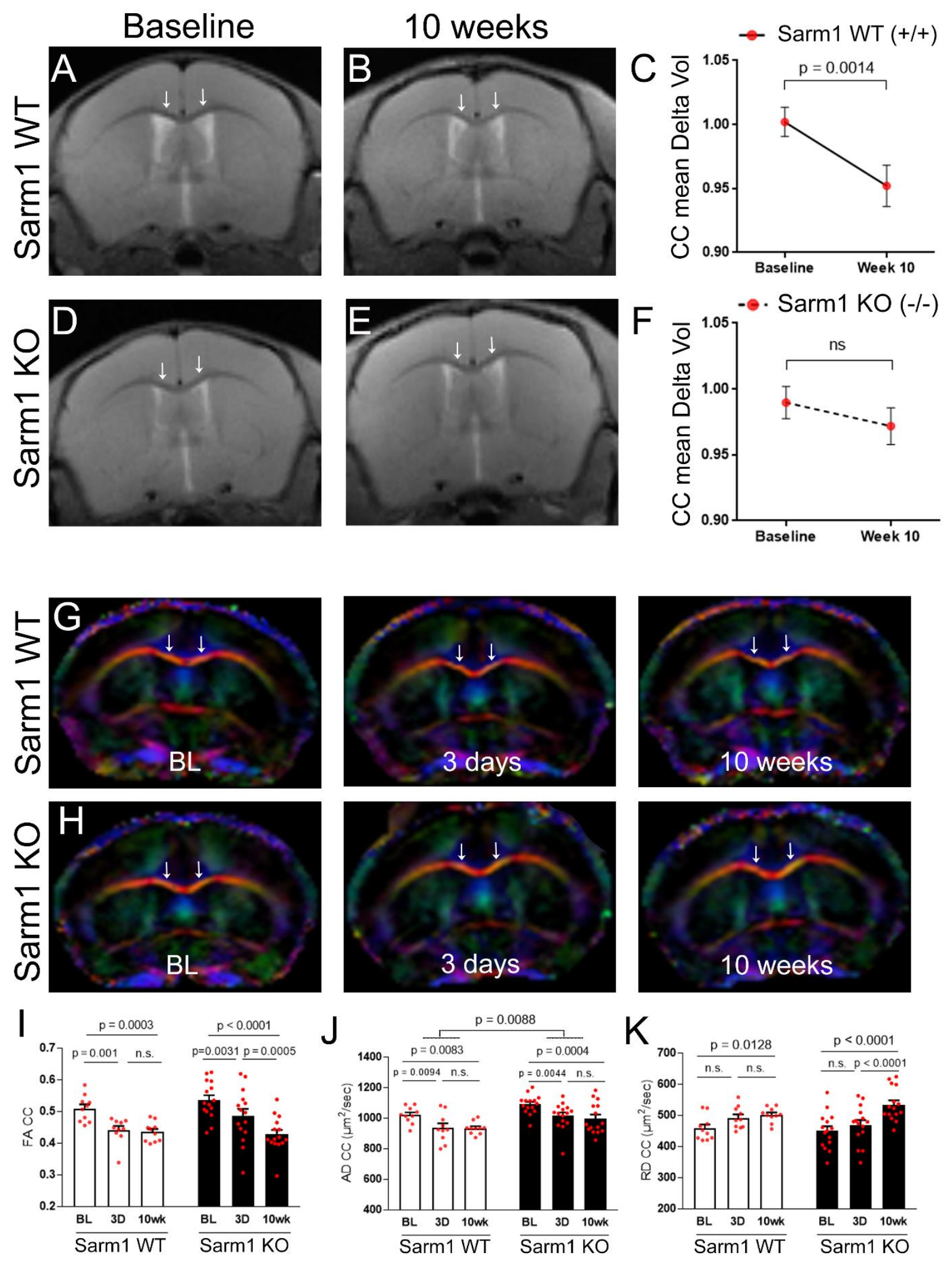

mice after sham or TBI procedures. Myelin is detected with immunolabeling for MOG (red). DAPI nuclear stain is shown in blue. CC borders are indicated by dashed lines. E: Sarm1 deletion attenuates CC atrophy, which is quantified based on the CC width. F: TBI results in significant myelin loss as detected by reduced MOG immunoreactivity. Myelin loss after TBI is significantly reduced in Sarm1 KO mice compared to Sarm1 WT mice. Sarm1 WT: $\mathrm{n}=7$ sham, $\mathrm{n}=7$ TBI.

Sarm1 KO: $n=6$ sham, $n=8$ TBI. $n s=$ not significant. A-D, scale bars $=100 \mu \mathrm{m}$.
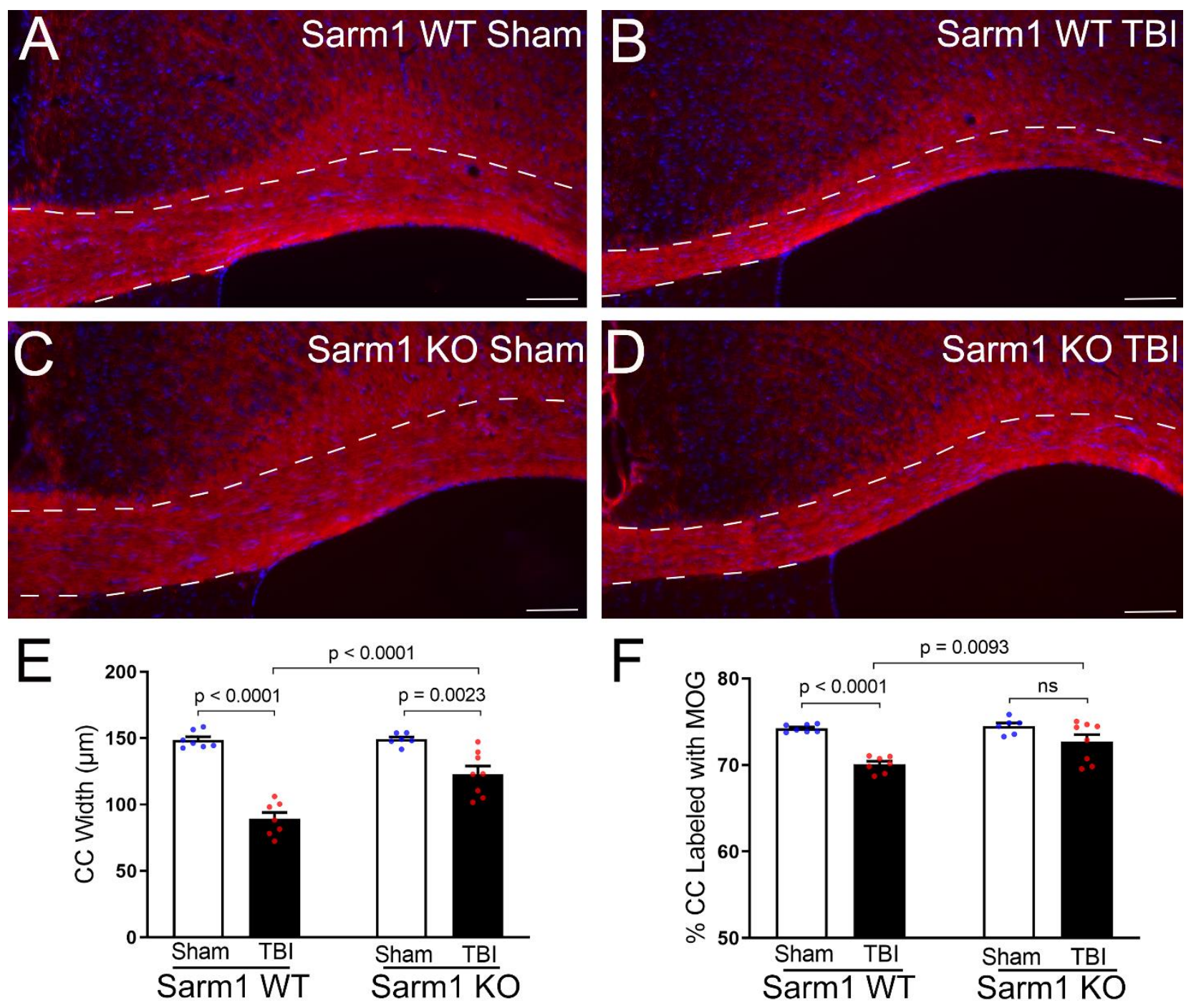
Figure 4. Sarm1 deletion reduces neuroinflammation at 10 weeks post-TBI. A-D:

920 Representative images from CC coronal sections of Sarm1 WT (A, B) and Sarm1 KO (C, D) mice

921 after sham $(\mathrm{A}, \mathrm{C})$ or $\mathrm{TBI}(\mathrm{B}, \mathrm{D})$ procedures. Neuroinflammation is detected with markers of

922 astrocytes (GFAP, green) and microglia (IBA1, red). DAPI nuclear stain shown in blue. The CC

923 borders are indicated by dashed lines. E-G: Higher magnification examples of astrocyte (GFAP)

924 and microglia (IBA1) morphology. In sham mice (E), astrocytes and microglia exhibit homeostatic

925 morphology with thin processes. Following TBI $(F, G)$, reactive astrocytes and microglia have

926 intensely immunolabeled cell bodies and shorter, thicker, processes. H: Sarm1 deletion

927 significantly reduced astrogliosis after TBI, based on GFAP immunolabeling within the CC area.

928 I: The microglial response also indicated CC neuroinflammation after TBI, based on IBA1

929 immunolabeling, but did not detect differences due to Sarm1 deletion. J-K: More detailed

930 counting of IBA1 immunolabeled (+) cells revealed that Sarm1 deletion significantly reduced the

931 frequency of both resting (J) and activated (K) microglia after TBI. Sarm1 WT: $\mathrm{n}=7$ sham, $\mathrm{n}=7$

932 TBI. Sarm1 KO: $n=6$ sham, $n=7$ TBI. $n s=$ not significant. A-D, scale bars $=100 \mu m$. E-G, scale

933 bars $=25 \mu \mathrm{m}$. 

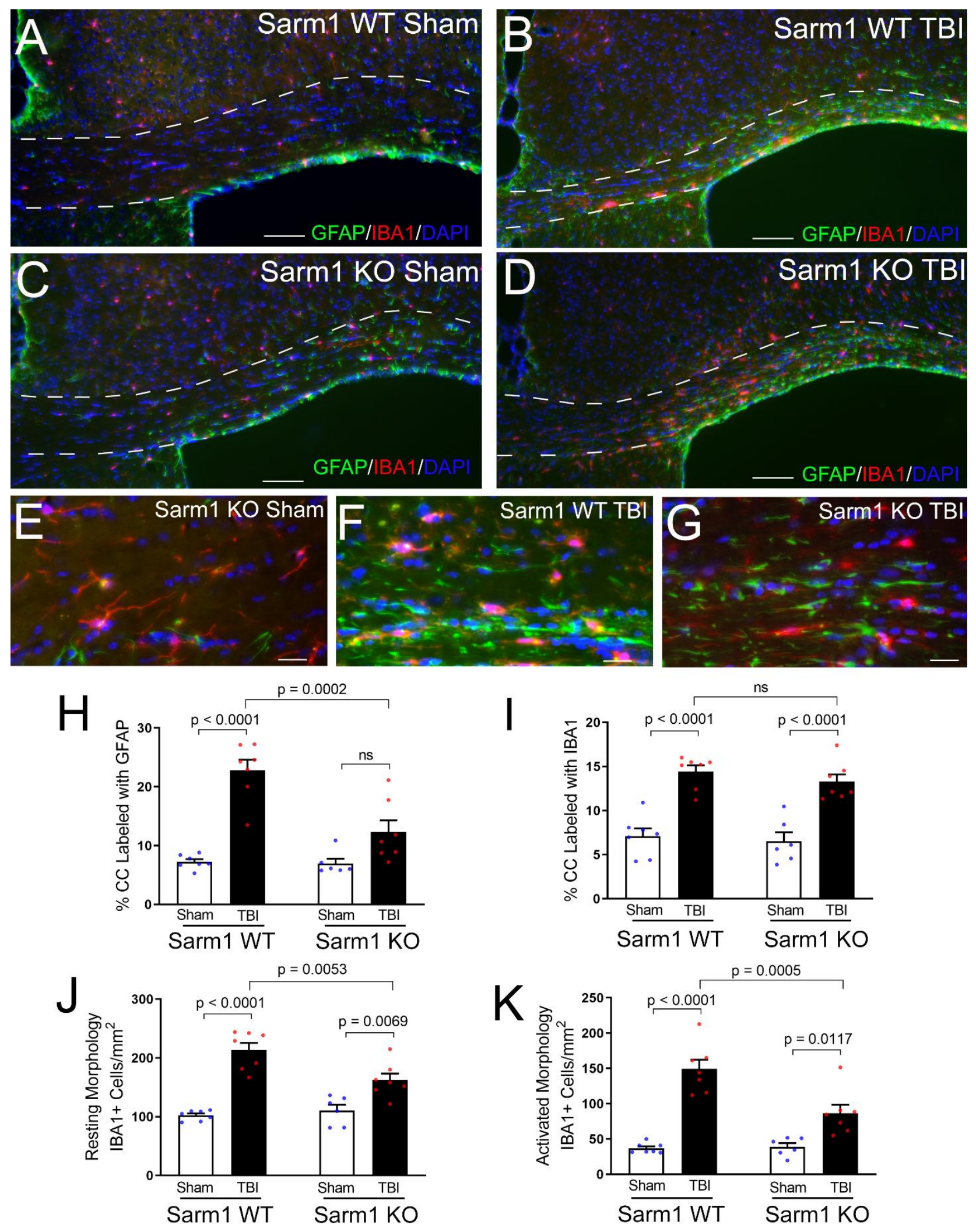
936 Figure 5. Sarm1 deletion improves motor learning in chronic stage TBI. Miss-step wheels

937 have irregularly spaced rungs to assess motor skill learning (week 1) followed by a plateau

938 phase (week 2) that tests bilateral sensorimotor function. A: Sarm1 KO mice more quickly learn

939 to run at a faster average velocity compared to Sarm1 WT mice, but this improvement does not

940 reach statistical significance $(p=0.0821)$. B: Sarm1 KO mice run on the wheels more frequently

941 than Sarm1 WT during the learning phase. C: Running behavior accumulates to increased total

942 distance traveled during the learning phase in Sarm1 KO mice compared to Sarm1 WT mice.

943 There were no statistically significant differences between genotypes in the three measures

944 during the plateau phase (A-C). Sarm1 WT: $n=9$ TBI. Sarm1 KO: $n=15$ TBI. $n s=$ not

945 significant. 

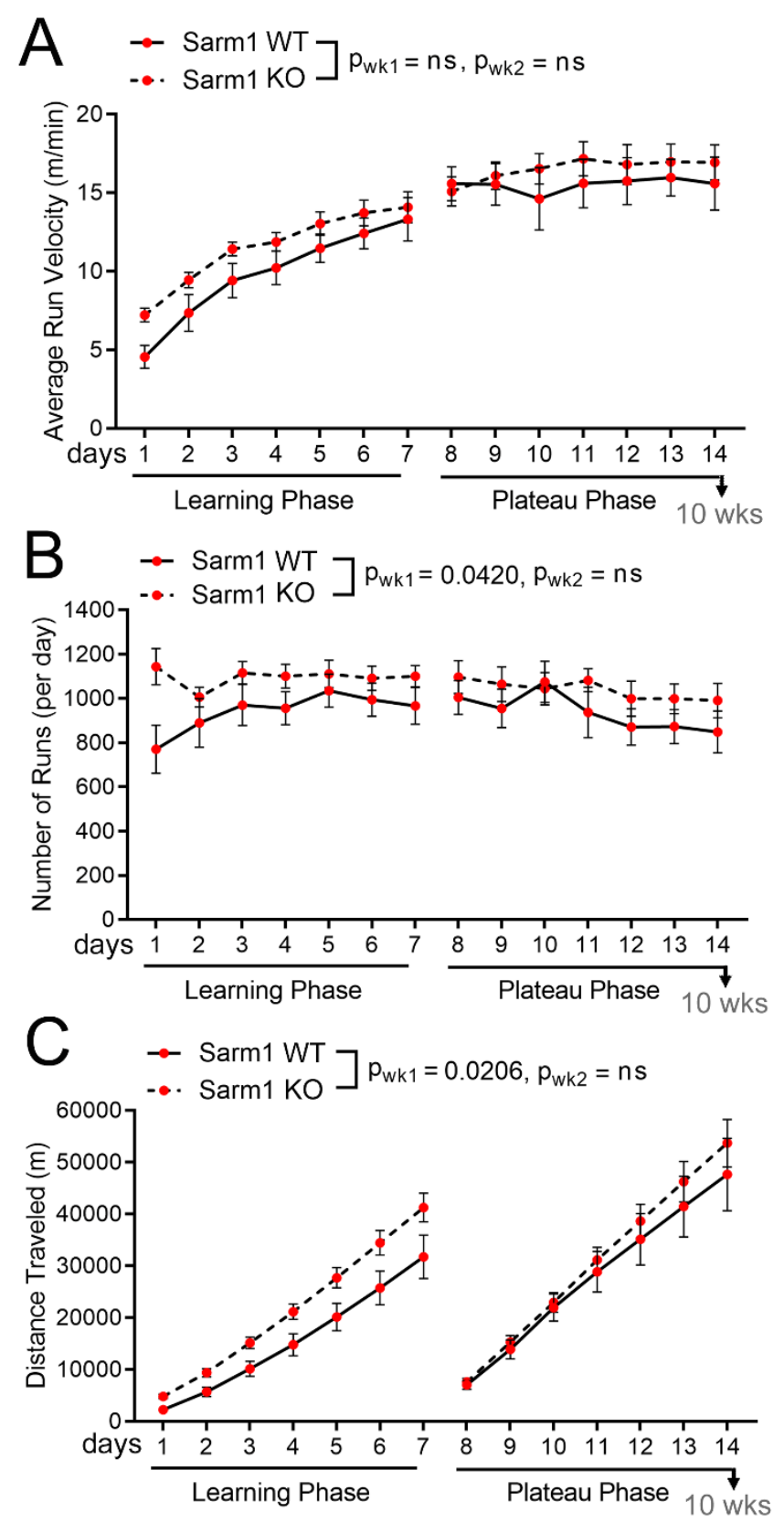

Figure 6. Sarm1 deletion normalizes time spent sleeping in the chronic stage of TBI: Mice were single housed in cages equipped with a PiezoSleep Mouse Behavioral Tracking System for $72 \mathrm{hrs}$ during the eighth week after TBI. A-B: Mice were acclimated for $18 \mathrm{hrs}$. Statistical

951 analysis focused on data collected during $48 \mathrm{hrs}$ across two complete cycles of lights on (white 952 bars) and lights off (dark bars and gray background). Recordings continued for a subsequent 6 
953 hrs to show the final wake/sleep transition. In Sarm1 WT mice (A), the time spent sleeping per

954 hour is significantly different between sham and TBI, with the injured mice appearing to sleep

955 less during the normal sleeping period when lights are on. In Sarm1 KO mice (B) the sleep

956 pattern was not different between sham and TBI conditions during the $48 \mathrm{hr}$ data collection

957 period. C: TBI significantly reduced the time spent sleeping for Sarm1 WT mice during the

958 normal sleeping period when lights are on. Sarm1 KO mice did not exhibit sleep loss after TBI.

959 D: During the dark period, sleep time was not different based on injury or genotype. Sarm1 WT:

$960 \mathrm{n}=8$ sham, $\mathrm{n}=10$ TBI. Sarm $1 \mathrm{KO}: \mathrm{n}=9$ sham, $\mathrm{n}=10$ TBI. $\mathrm{ns}=$ not significant. 


\section{A Sarm1 WT}

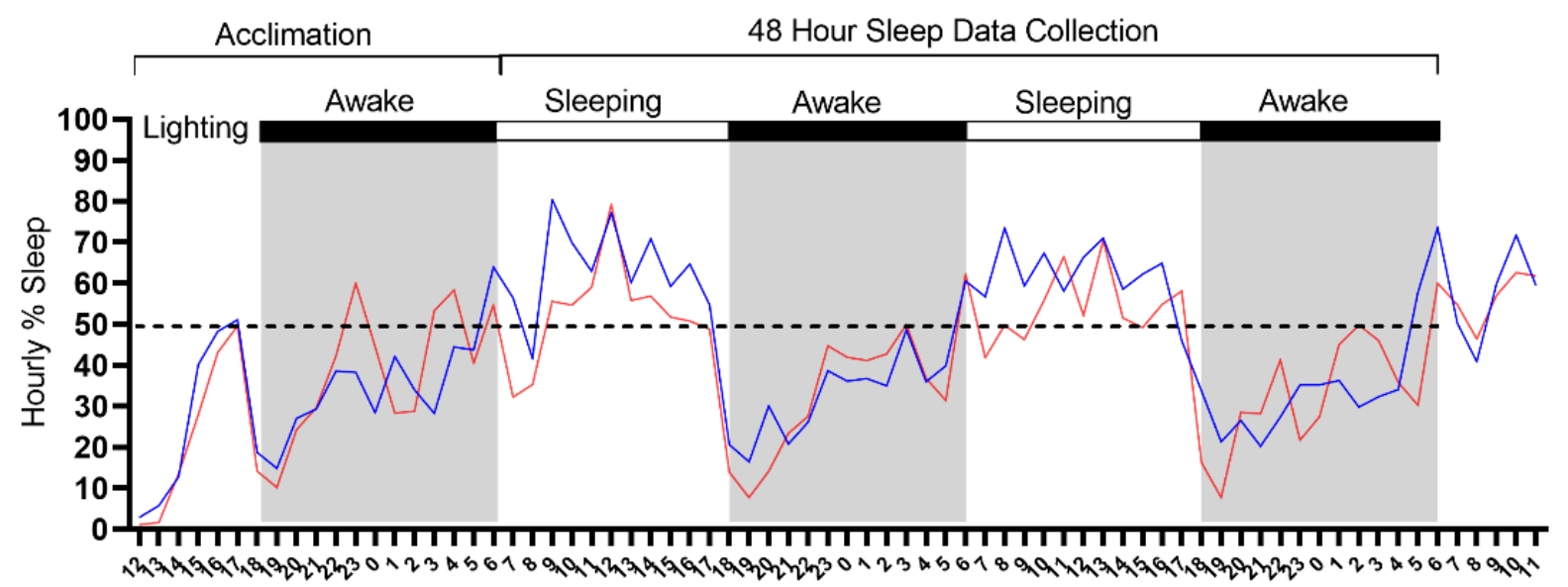

Time of Day

\section{B Sarm1 KO}

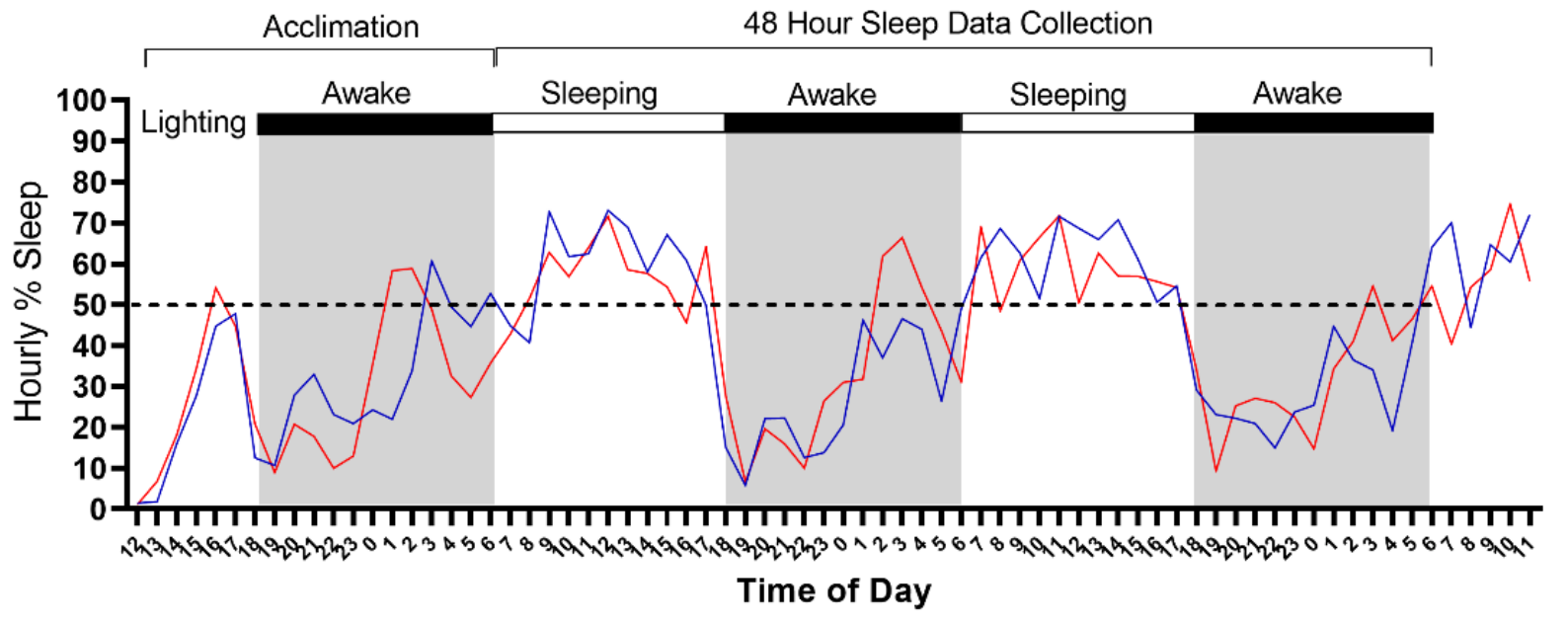

\section{Lights ON (sleep phase)}

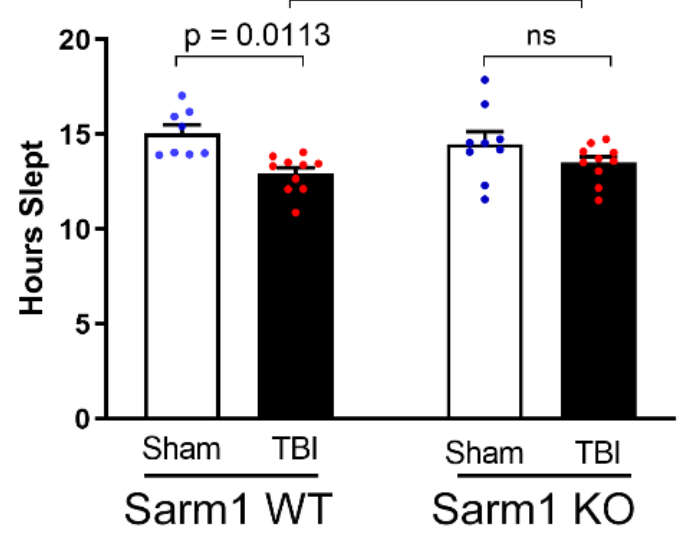

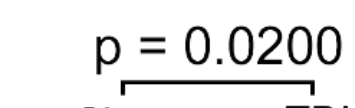

- Sham - TBI

48 Hour Sleep Data Collection 


\title{
Genetic inactivation of SARM1 axon degeneration pathway improves outcome trajectory after experimental traumatic brain injury based on pathological, radiological and functional measures
}

\author{
Donald V. Bradshaw Jr.1,2, Andrew K. Knutsen ${ }^{3,4}$, Alexandru Korotcov,4, Genevieve M. \\ Sullivan², Kryslaine L. Radomski ${ }^{2,4}$, Bernard J. Dardzinski ${ }^{3,4}$ *, Xiaomei Zi², Dennis P. McDaniel ${ }^{5}$, \\ and Regina C. Armstrong ${ }^{1,2,4}$ \#
}

${ }^{1}$ Graduate Program in Neuroscience,

${ }^{2}$ Department of Anatomy Physiology and Genetics

${ }^{3}$ Department of Radiology and Radiological Sciences,

${ }^{4}$ Center for Neuroscience and Regenerative Medicine,

${ }^{5}$ Biomedical Instrumentation Center,

F. Edward Hebert School of Medicine, Uniformed Services University of the Health Sciences, Bethesda, MD 20814 USA

\section{\#Corresponding Author:}

Regina C. Armstrong, PhD

Department of Anatomy, Physiology and Genetics

Uniformed Services University of the Health Sciences

4301 Jones Bridge Rd., Bethesda, MD 20814

E-mail: regina.armstrong@usuhs.edu

${ }^{*}$ Current address:

Center for Scientific Review

National Institutes of Health, Bethesda, MD

\section{Supplemental Figure S1: Righting reflex times after sham or TBI surgical procedure in}

Sarm1 WT and Sarm1 KO mice. Post-surgical righting reflex times were measured as the time interval from the end of anesthesia until mice returned to the upright position. Righting time is shown for all mice used in experiments. Prolonged righting reflex times are an indicator of TBI severity that serve as a measure of arousal that estimates loss of consciousness after TBI or sham procedures. Righting reflex time did not differ between male and female littermates, so the sexes were combined for analysis by genotype. Righting reflex time was significantly prolonged after TBI as compared to the sham procedure for Sarm1 WT $(\mathrm{p}<$ $0.0001)$ and Sarm1 KO ( $p<0.001)$ mice without a difference based on genotype ( $p=0.8287$; Two-way ANOVA; $F(1,68)=183)$.

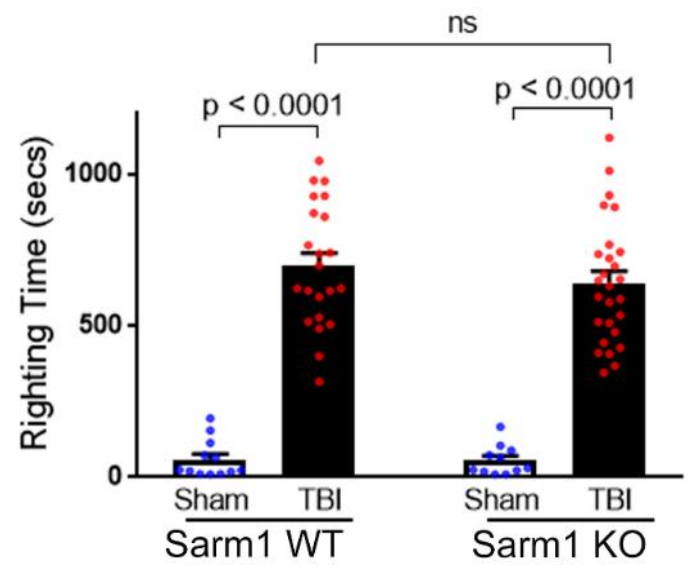




\section{Supplemental Figure S2. TBI reduces white matter integrity and produces significant} corpus callosum (CC) atrophy in C57BL/6 mice. A cross-sectional in vivo MRI study used male C57BL/6J mice between 9-10 weeks post-TBI/sham. After scanning, mice were perfused for tissue analysis at 10 weeks post-TBI/sham. A-B: CC volume changes were calculated as the change in volume compared to a registered template image of all animals in the study. Seven ROls were manually drawn on coronal images (125 $\mu$ m thickness) encompassing the rostrocaudal CC over the lateral ventricle and underneath the impact site at bregma (A). TBI significantly reduced the mean $\mathrm{CC}$ volume, as compared to the sham procedure (B) C-E: Postimaging neuropathology validated CC atrophy after TBI. Myelinated fibers were immunolabeled for myelin oligodendrocyte glycoprotein (MOG; red) and cellular distribution was detected using nuclear counterstain (DAPI; blue) (C, D). The CC borders (dashed lines) were evident by the pattern of myelinated fibers oriented in the medial-lateral direction in the $\mathrm{CC}$ in contrast to the myelinated fibers of the cingulum $(\mathrm{Cg})$ that align in the rostro-caudal (i.e. anterior-posterior) direction. Double headed arrows (C, D) show examples of sites for CC width measurement for quantification (E). F-J: MRI diffusion tensor imaging (DTI) illustrates fractional anisotropy signal in the CC (white arrows) and adjacent regions in direction encoded color maps (F, G). Colors represent fiber directions as red (medial-lateral), blue (anterior-posterior), and green (superiorinferior). TBI significantly reduced the CC fractional anisotropy (FA; $H$ ) which was driven by reduced axial diffusivity $(A D ; I)$ and increased radial diffusivity $(R D ; J) . M R I n=12$ per condition; male C57BL/6J mice (RRID:IMSR_JAX:000664), Jackson Laboratories, Bar Harbor, ME). Neuropathology $\mathrm{n}=6$ per condition randomly selected from the MRI cohort. Student's $t$-test.

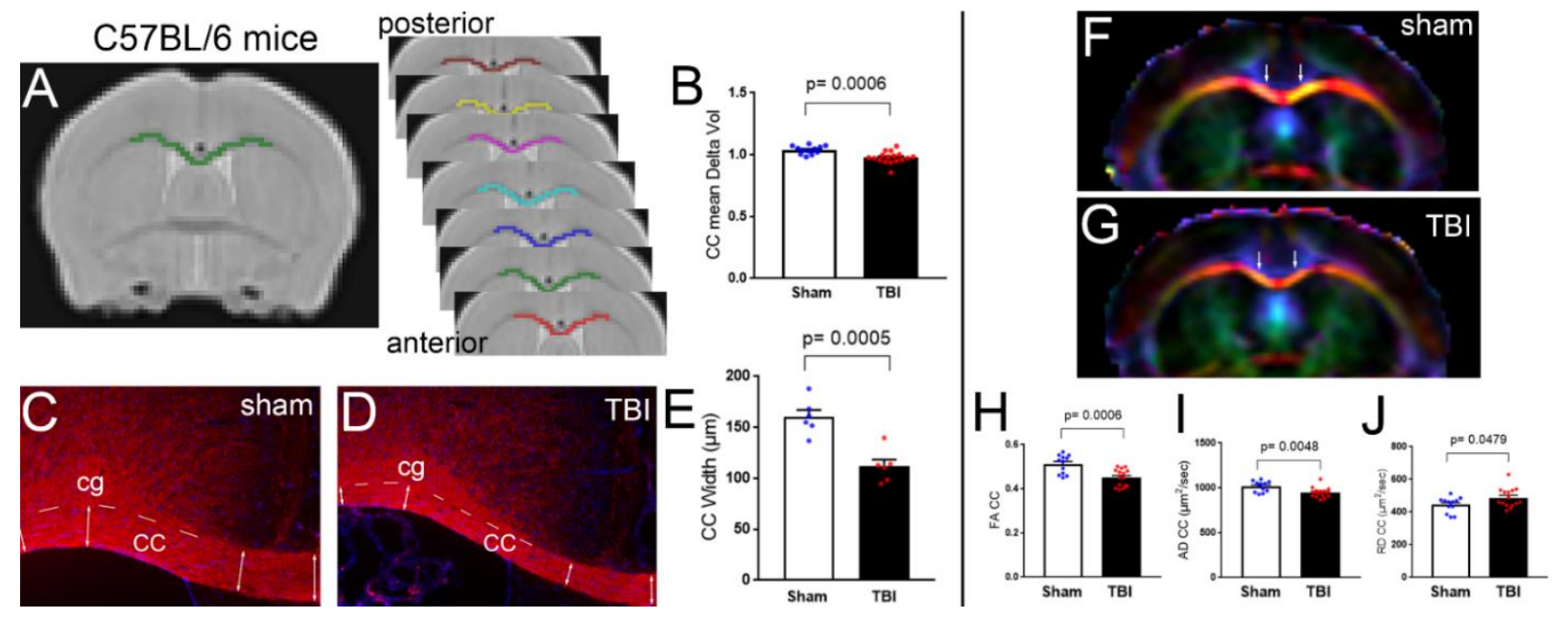




\section{Supplemental Figure S3. TBI causes a chronic stage motor learning deficit in C57BL/6J}

mice. Miss-step wheels, which have been shown to engage the CC and be sensitive to myelination status, were used to assess chronic stage changes between sham and TBI mice. The wheels have irregularly spaced rungs to assess motor skill learning (week 1) followed by a plateau phase (week 2) that tests bilateral sensorimotor function. TBI mice show a significant decrease in average running velocity compared to sham during the learning phase (week 1). There was no statistically significant difference between TBI and sham groups during the plateau phase of the assay (week 2). Wheels $n=9$ per condition; male C57BL/6J mice (RRID:IMSR_JAX:000664), Jackson Laboratories, Bar Harbor, ME). Two-way repeated measures mixed effects model ANOVA; Learning Phase: Time $p<0.0001, F(2.640,40.93)=$ 84.41; Injury $p=0.0374, F(1,16)=5.151$; Time $x$ Injury $p=0.0299, F(6,93)=2.457$. Plateau Phase: not significant.

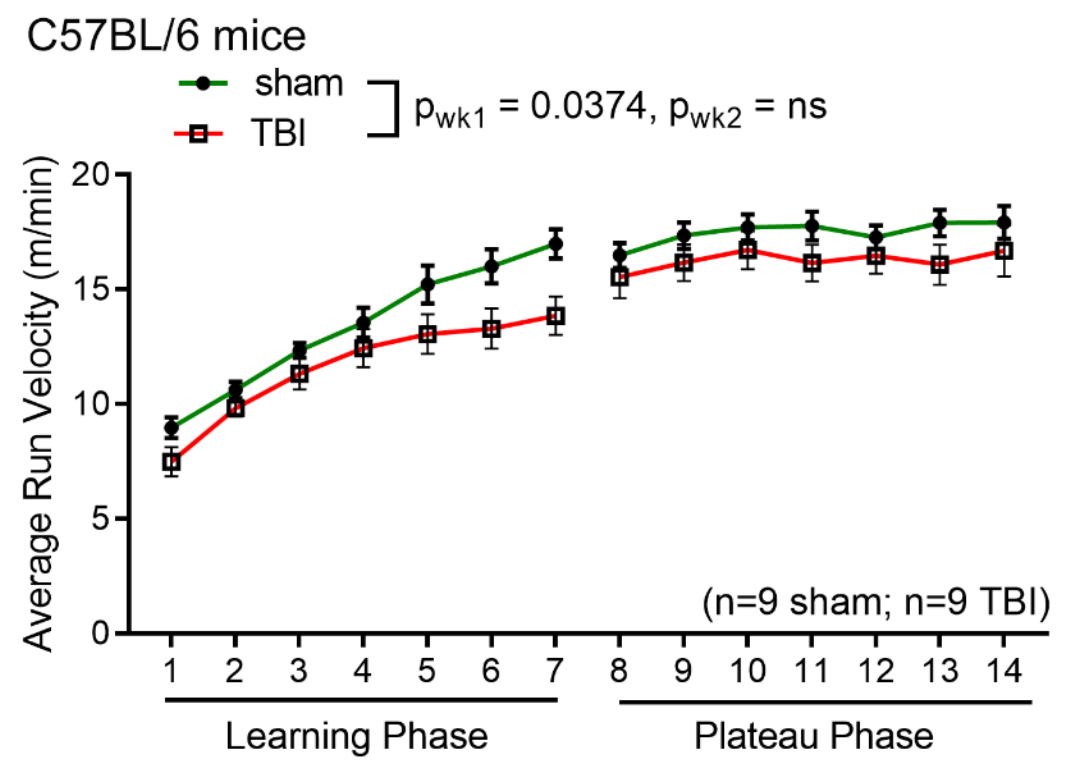

\section{Supplemental Figure S4. TBI does not induce a late-stage social deficit despite CC}

atrophy in C57BL/6J mice. In their $8^{\text {th }}$ week post injury mice were tested using a social interaction assay to measure propensity to engage in social behavior. For five minutes mice freely explore a three chamber apparatus with one chamber containing a novel, unfamiliar mouse in a wire carrier and another possessing an empty wire carrier while their time spent interacting with each carrier is measured. Our previous study showed that CC pathology in an 
experimental model of chronic demyelination induces a social deficit [1]. This study looked to determine if a social deficit, that was not present at more acute time points after single impact TBI [2], develops at a chronic time point. Both TBI and sham mice spent a significantly greater amount of time interacting with the carrier containing the unfamiliar mouse compared to the empty carrier (A). There was no statistically significant difference in interaction time between sham and TBI groups. At 10 weeks post TBI mice were sacrificed for neuropathological evaluation of CC width. TBI mice exhibit significant late-stage CC atrophy compared to sham (B). The absence of a social deficit at 8 weeks post TBI despite ongoing, chronic atrophy of the $\mathrm{CC}$ resulted in the omission of the social interaction assay from our study evaluating the therapeutic benefit of Sarm1 genetic deletion. Social Interaction $n=12$ per condition; male C57BL/6J mice (RRID:IMSR_JAX:000664), Jackson Laboratories, Bar Harbor, ME).

Neuropathology $n=6$ per condition randomly selected from the MRI cohort. Social interaction:

Two-way ANOVA; $F(1,44)=0.2116$. Neuropathology: Student's $t$-test.

A

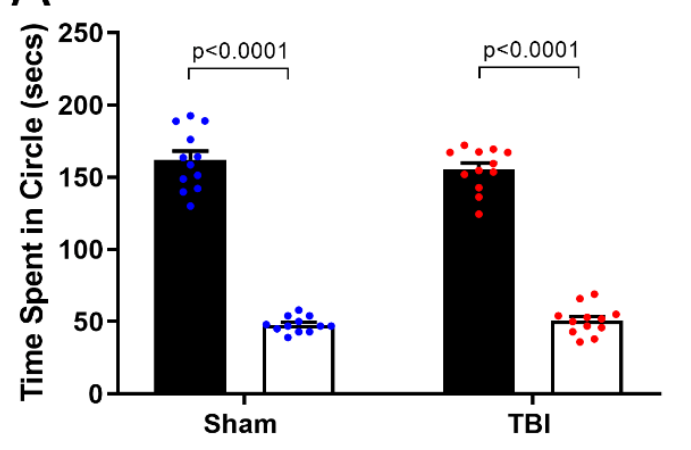

C57BL/6 mice

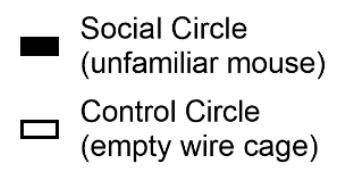

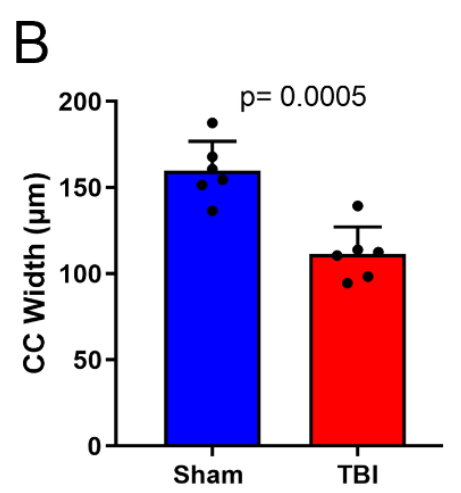

\section{REFERENCES}

1 Sullivan GM, Knutsen AK, Peruzzotti-Jametti L, Korotcov A, Bosomtwi A, Dardzinski BJ, Bernstock JD, Rizzi S, Edenhofer F, Pluchino Set al (2020) Transplantation of induced neural stem cells (iNSCs) into chronically demyelinated corpus callosum ameliorates motor deficits. Acta Neuropathol Commun 8: 84 Doi 10.1186/s40478-020-00960-3

2 Yu F, Shukla DK, Armstrong RC, Marion CM, Radomski KL, Selwyn RG, Dardzinski BJ (2017) Repetitive Model of Mild Traumatic Brain Injury Produces Cortical Abnormalities Detectable by Magnetic Resonance Diffusion Imaging, Histopathology, and Behavior. J Neurotrauma 34: 13641381 Doi 10.1089/neu.2016.4569 